\title{
Effects of Attraction of Vacuum Extractors of Different Materials and Pressures on the Fetal Head During Delivery
}

\section{Yu-Hsuan Chen}

National Defense Medical Center

Kuo-Min Su

National Defense Medical Center

Ming-Tzu Tsai

HungKuang University

Chi-Kung Lin

National Defense Medical Center

Cheng-Chang Chang

National Defense Medical Center

Kuo-Chih Su ( $\nabla$ kcsu@vghtc.gov.tw)

Taichung Veterans General Hospital

\section{Research Article}

Keywords: Vacuum extractor, Attractive pressures, Operative delivery, Biomechanics, Finite element analysis

Posted Date: June 1st, 2021

DOl: https://doi.org/10.21203/rs.3.rs-543589/v1

License: (9) This work is licensed under a Creative Commons Attribution 4.0 International License. Read Full License 


\section{Abstract}

\section{Purpose}

In some cases where operative deliveries are required with vacuum extractor, and obstetricians could choose the vacuum extractor to facilitate the process smoother and safer. However, there is no related biomechanical literature about the influences of vacuum extractors fabricated from different materials and pressures of vacuum on the fetal head. Hence, we utilized the finite element method to investigate the influences of vacuum extractors manufactured from different materials on the fetal head under various extractive pressures.

Methods

First, the finite element analysis models of vacuum extractor and fetal head were established. The vacuum extractor model was designed as a hemispherical shape and we compared silicone rubber and stainless steel for the materials of vacuum extractor. Subsequently, four different vacuum pressures were applied as the factors for investigation-500- $\mathrm{cm} \mathrm{H}_{2} \mathrm{O}, 600-\mathrm{cm} \mathrm{H}_{2} \mathrm{O}, 700-\mathrm{cm} \mathrm{H}_{2} \mathrm{O}$, and 800-cm $\mathrm{H}_{2} \mathrm{O}$. Finally, we observed and analyzed the reactive force on the fetal head, von Mises stress of vacuum extractor, and von Mises stress on the skull of fetal head to evaluate the influences of vacuum extractors of different materials under different pressures.

Results

The results demonstrated that different vacuum pressures had only a slight difference of influences on the fetal head. The use of stainless-steel vacuum extractors caused a relatively larger reactive force $(358.04-361.37 \mathrm{~N})$ and stress (13.547-13.675 MPa) on the fetal head.

\section{Conclusions}

Non-metallic or relatively softer materials could be selected when using a vacuum extractor for operative delivery to avoid complications such as scalp scratch, and even cephalohematoma and intracerebral hemorrhage.

\section{Introduction}

Operative delivery is a lifesaving technique in delivery units, wherein obstetrical physicians can use vacuum extractor and forceps to help for a smoother and safer delivery [1]. However, using these tools to assist in vaginal delivery still has related risks. Taking vacuum extractor as an example, mothers may have a relatively high chance of experiencing fourth-degree perineal lacerations [2]. Fetuses would have higher chance of experiencing caput saccucedeum, cephalohematoma, subgaleal hematoma, scalp laceration, and even intracranial hemorrhage [3]. 
Currently, in accordance with the clinical demands, vacuum extractors with a great variety of designs fabricated from different materials are available on the market [4]. Vacuum extractors are made of different materials such as soft (e.g., silicone rubber) and hard (e.g., metals) materials [5]. According to clinical experience of application, vacuum extractors made of soft materials can easily to use and infants are less likely to be injured compared with vacuum extractors of metal materials for extraction [6]. Moreover, if the vacuum pressure is too high, the use of the vacuum extractor may still cause injuries to newborns [7]. Although the above is known clinically, there is still no related biomechanical research that can clearly indicate the influences of vacuum extractors manufactured from different materials and at different vacuum pressures.

In clinical practice, observing the force on the fetus exerted by the vacuum extractor under the attractive pressure on the fetal head during the delivery process is quite difficult. Therefore, some scholars used finite element analysis to evaluate the influences on the fetal head during delivery in the past [8]. For example, observation of the distribution of pressure on the fetal head at the first stage of labor and analysis of the biomechanical effects of placing the vacuum extractor in different positions were all performed by way of finite element analysis and even the finite element analysis could be used to evaluate the effects of using forceps with different angles on the fetal head [9-11]. Therefore, although exploring the influences of external force on the fetal head during operative delivery with vacuum extractor in clinical practice is not easy to implement, the method of finite element analysis can be made use of simulating the real clinical situation and exploring the influences of attractive force on the fetal head in the delivery process.

Based on previous literature, though there have been several studies on biomechanical analysis of forceps or vacuum extractors during delivery, there is still no further biomechanical evaluation on the materials of vacuum extractors or vacuum pressures so far. Therefore, in this study, we mainly aim to use the finite element method to investigate the influences of vacuum extractors made of different materials on the fetal head under extraction of various vacuum pressures. The results of this study could provide a biomechanical basis as a crucial reference for clinicians to reduce the incidence of fetal head injuries and other complications in while choosing and using vacuum extractors during delivery.

\section{Materials And Methods}

\subsection{Building a simulation geometry model}

In this study, to investigate the influences of using different materials of vacuum extractors and different vacuum pressures, finite element models of vacuum extractor and fetal head were established, and the design of vacuum extractor model was mainly hemispherical. According to previous literature [12], a cup of 5 -cm diameter is suitable for nearly all deliveries, so the vacuum extractor model used herein was a hemispherical vacuum extractor with a diameter of $5 \mathrm{~cm}$ (Fig. 1). This three-dimensional (3D) model of vacuum extractor was plotted using 3D computer graphics software SolidWorks (SolidWorks 2016, Dassault Systems SolidWorks Corp, Waltham, MA, USA). In addition, because the effect of vacuum 
extractor on the fetal head was to be evaluated, a fetal head model was established. The fetal head model was a computer model established mainly based on previous research [11]. The fetal head computer model was mainly established by referring to the fetal head anatomical model. In addition, the fetal head was divided into the structures of scalp and skull, which were a 1-mm-thick scalp and 2-mmthick skull, respectively. Therefore, the computer models used herein were mainly divided into three parts, namely the fetal head scalp, fetal head skull, and vacuum extractor (Fig. 1). The established model was imported into finite element analysis software ANSYS Workbench (ANSYS Workbench 18.0, ANSYS, Inc., Canonsburg, PA) for finite element analysis.

\subsection{Loading conditions and boundary conditions}

In this study, we mainly simulated the force exerted by different vacuum extractors and different vacuum pressures on the fetal head. Therefore, in this study, we would adopt a boundary condition and two different load conditions. The first load condition was to simulate the vacuum pressure in the fetal head area that provides head extraction force when the vacuum extractor extracts the fetal head. According to the previous literature [12], the operating vacuum pressure near all ventouses is $500-800-\mathrm{cm} \mathrm{H} 20(0.6-$ $0.8 \mathrm{~kg} / \mathrm{cm} 2 ; 60-80 \mathrm{kPa}$ ). To explore the influences of different vacuum pressures, four different vacuum pressures were selected as the factors to be investigated-500- $\mathrm{cm} \mathrm{H}_{2} \mathrm{O}, 600-\mathrm{cm} \mathrm{H} \mathrm{H}_{2} \mathrm{O}, 700-\mathrm{cm} \mathrm{H}_{2} \mathrm{O}$, and $800-\mathrm{cm} \mathrm{H}_{2} \mathrm{O}$. The area applied with the boundary condition setting of vacuum pressure was mainly the inner side of vacuum extractor (Fig. 2(a)). The second load condition simulates the situation of pulling outward by the vacuum extractor. The operation mode herein applies a 1-mm displacement in the $Y$ direction to the end of the vacuum extractor using displacement control (Fig. 2 (b)). In addition, as for the boundary conditions herein, the neck of fetal head was set as a fixed area (the area marked with green color in Fig. 1), and the displacement of $X$ axis, $Y$ axis, and $Z$ axis in this area was set as 0.

\subsection{Material properties of the model}

The research model comprised three parts, namely the scalp of fetal head, skull of fetal head, and vacuum extractor. The materials used for the vacuum extractor were mainly silicone rubber and stainless steel, which are commonly used clinically. The defined properties of materials used herein were mainly obtained from previous studies by other scholars [13-15]. All materials would be assumed to be homogeneous, isotropic, and linear elastic. Therefore, two independent parameters (Young's modulus and Poisson's ratio) would be used to express the properties of materials. Table 1 shows the values defined for the properties of materials used in the simulation herein. In addition, the mesh element used in the computer model of finite element analysis herein was a tetrahedral mesh (Fig. 3). After the convergence test, the model reached the $5 \%$ stop criteria of convergence test [16], so the finite element mesh model used in this study is reasonable (the mesh size used herein was $4 \mathrm{~mm}$ ). The computer finite element analysis model adopted herein had 131,198 nodes and 65,270 elements. 
Table 1

Material properties setting in this study [13-15].

\begin{tabular}{|lll|}
\hline Material & Young's modulus (MPa) & Poisson's ratio \\
\hline Scalp & 16.7 & 0.42 \\
\hline Skull & 2500 & 0.22 \\
\hline Silicone rubber & 10.3 & 0.49 \\
\hline stainless steel & 200000 & 0.3 \\
\hline
\end{tabular}

After the finite element analysis, the main observation indices herein were reaction force at the fixed end, von Mises stress on vacuum extractor, and von Mises stress on fetal head skull (among which the value of von Mises stress was defined as

$$
\left.\sigma_{v o n}=\sqrt{\frac{1}{2}\left[\left(\sigma_{1}-\sigma_{2}\right)^{2}+\left(\sigma_{1}-\sigma_{3}\right)^{2}+\left(\sigma_{2}-\sigma_{3}\right)^{2}\right]}\right)
$$

where $\sigma_{1}, \sigma_{2}$, and $\sigma_{3}$ represent the principal stress along the three axes). According to these observation indices, the biomechanical effects of vacuum extractors fabricated from different materials and at different vacuum pressures on the fetal head could be investigated.

\section{Results}

In this study, after the finite element analysis, the reaction force as well as the corresponding stress distribution on the vacuum extractor and fetal skull with the neck of fetal head as the fixed end could be obtained. Figure 4 shows the numerical magnitude and stress direction of the reaction force on the fetal head with different materials of vacuum extractors and under different vacuum pressures. Table 2 shows the magnitude of reaction force and its components in each axis. With the increase in vacuum pressure, the magnitude of reaction force would increase slightly. In addition, when using vacuum extractors fabricated from different materials, the numerical values of reaction force would differ significantly. On the whole, when silicone rubber was used as the material of vacuum extractor $(12.229-15.064 \mathrm{~N})$, the reaction force would be smaller compared with using stainless steel (358.04-361.37 N). 
Table 2

Magnitudes and components of reaction force

\begin{tabular}{|c|c|c|c|c|c|}
\hline & & $500-\mathrm{cm} \mathrm{H}_{2} \mathrm{O}$ & $600-\mathrm{cm} \mathrm{H}_{2} \mathrm{O}$ & $700-\mathrm{cm} \mathrm{H}_{2} \mathrm{O}$ & $800-\mathrm{cm} \mathrm{H}_{2} \mathrm{O}$ \\
\hline \multirow[t]{4}{*}{ Silicone rubber } & $X$ Axis & $9.4194 \times 10^{-5} \mathrm{~N}$ & $1.1303 \times 10^{-4} \mathrm{~N}$ & $1.3187 \times 10^{-4} \mathrm{~N}$ & $1.5071 \times 10^{-4} \mathrm{~N}$ \\
\hline & Y Axis & $-12.229 \mathrm{~N}$ & $-13.174 \mathrm{~N}$ & $-14.119 \mathrm{~N}$ & $-15.064 \mathrm{~N}$ \\
\hline & Z Axis & $9.1197 \times 10^{-5} \mathrm{~N}$ & $1.0944 \times 10^{-4} \mathrm{~N}$ & $1.2768 \times 10^{-4} \mathrm{~N}$ & $1.4592 \times 10^{-4} \mathrm{~N}$ \\
\hline & Total & $12.229 \mathrm{~N}$ & $13.174 \mathrm{~N}$ & $14.119 \mathrm{~N}$ & $15.064 \mathrm{~N}$ \\
\hline \multirow[t]{4}{*}{ stainless steel } & $X$ Axis & $9.4307 \times 10^{-5} \mathrm{~N}$ & $1.1315 \times 10^{-4} \mathrm{~N}$ & $1.3199 \times 10^{-4} \mathrm{~N}$ & $1.5082 \times 10^{-4} \mathrm{~N}$ \\
\hline & Y Axis & $-358.04 \mathrm{~N}$ & $-359.15 \mathrm{~N}$ & $-360.26 \mathrm{~N}$ & $-361.37 \mathrm{~N}$ \\
\hline & Z Axis & $9.1211 \times 10^{-5} \mathrm{~N}$ & $1.0945 \times 10^{-4} \mathrm{~N}$ & $1.2769 \times 10^{-4} \mathrm{~N}$ & $1.4593 \times 10^{-4} \mathrm{~N}$ \\
\hline & Total & $358.04 \mathrm{~N}$ & $359.15 \mathrm{~N}$ & $360.26 \mathrm{~N}$ & $361.37 \mathrm{~N}$ \\
\hline
\end{tabular}

Figure 5 mainly shows the distribution of von Mises stress on vacuum extractors with different materials of vacuum extractors and under different vacuum pressures. The results showed that greater the vacuum pressure applied, the greater is the von Mises stress on the vacuum extractor. Besides, when different materials were used for the vacuum extractor, the magnitude of von Mises stress on the vacuum extractor would differ significantly. When the material used for the vacuum extractor was silicone rubber (0.5858$0.9510 \mathrm{MPa}$ ), the von Mises stress on the vacuum extractor would be smaller compared with using stainless steel.

Figure 6 shows the von Mises stress distribution on the fetal head skull measured under different vacuum extractor forces. The results showed that when silicone rubber was used as the material of vacuum extractor, the von Mises stress in the area on the fetal head skull extracted by the vacuum extractor would show relatively great stress with the increase in vacuum pressure. Furthermore, the maximum stress occurred near the center of the sphere part on the vacuum extractor. In addition, when stainless steel was used as the material of vacuum extractor, the von Mises stress on the vacuum head skull increased slightly with the increase in vacuum pressure; moreover, there was high stress distribution on the vacuum head skull in the outer area of the vacuum extractor. When silicone rubber (2.4755-4.0156 MPa) was used as the material of vacuum extractor, the value of von Mises stress on the fetal head skull would be smaller compared with using stainless steel (13.547-13.675 MPa).

\section{Discussion}

In this study, we successfully performed finite element analysis to explore the influences of vacuum extractors fabricated from different materials and under different vacuum pressures on the fetal head during the delivery process. Up to this time, there is no detailed mechanical basis for investigating the influences of using vacuum extractors made from different materials in clinical practice. The results of 
this study could provide a reliable reference basis for clinical obstetricians for choosing the material and magnitude of pressure of vacuum extractor when they need to use for operative delivery. In addition, based on these results, the complications including head deformation, scalp abrasion, even cephalohematoma, and intracerebral hemorrhage caused by the vacuum extractor during operative vaginal deliveries could also be reduced and alleviated.

According to the observation of reaction force herein, when the vacuum extractor was made of stainless steel, the fixed end of fetal head neck was subjected to a relatively large reaction force. The main reason can be explained by the content put forward in the textbook Mechanics of Materials [17]. When $F=\sigma A$ and $\sigma=E \varepsilon$, where $\sigma$ is the stress, $F$ is the external force, $A$ is the cross-sectional area, $E$ is the Young's modulus, and $\varepsilon$ is the strain. Therefore, it can also be expressed as $F=E \varepsilon A$. When the displacement changes given are consistent and the stressed section is the same, the external force will be proportional to the Young's modulus. Therefore, as the Young's modulus of stainless steel used in this study was $200,000 \mathrm{MPa}$ and that of silicone rubber was 10.3 MPa, stainless-steel vacuum extractors would make the fixed end of fetal head neck subject to a relatively large reaction force. In addition, by observing the influences of different vacuum pressures on the reaction force on the fixed end of fetal head neck, when a greater vacuum pressure was applied, the reaction force on the fixed end of fetal head neck would increase slightly. According to the textbook Mechanics of Materials [17], the mechanical analysis description for spherical shells indicates that $F=\sigma(2 \pi r t)=p\left(\pi r^{2}\right)$, where $\sigma$ is the stress on the spherical shell, $r$ is the inner radius of spherical shell, $p$ is the vacuum pressure, and $t$ is the wall thickness on the vacuum extractor. Hence, the reaction force on the fixed end of fetal head neck is proportional to the vacuum pressure. The greater the vacuum pressure, the greater is the reaction force on the fixed end of fetal head neck.

By observing the stress on the vacuum extractor, the greater the vacuum pressure, the greater is the von Mises stress on the vacuum extractor. Because $\sigma(2 \pi r t)=p\left(\pi r^{2}\right)$, the stress on the spherical shell of vacuum extractor was $\sigma=\mathrm{pr} / 2 \mathrm{t}$. Therefore, when the vacuum extractor had a fixed shape, $r$ and $t$ in the formula were fixed values, so the stress on the vacuum extractor was proportional to the vacuum pressure. Hence, the greater the vacuum pressure applied, the greater is the von Mises stress on the vacuum extractor. In addition, when different materials were used for the vacuum extractor, the von Mises stress on the vacuum extractor was based on $\sigma=E \varepsilon$, and when the given displacement was the same, stress $(\sigma)$ would be proportional to the Young's modulus (E). Therefore, using stainless steel as the material of vacuum extractor would cause relatively high stress (47.517-48.385 MPa) on the vacuum extractor. Although using stainless steel would have higher stress compared with using silicone rubber, the yield stress value of stainless steel is approximately $700 \mathrm{MPa}$ [18]. In this study, when stainless steel was used as the material of vacuum extractor, the result of stress value on the vacuum extractor was much lower than $700 \mathrm{MPa}$. Therefore, using stainless steel as the material of vacuum extractor would not cause permanent deformation of the vacuum extractor, while using silicone rubber as the material is prone to deformation. 
In addition, by observing the stress on the simulated fetal head skull structure, when silicone rubber was used as the vacuum extractor material, the stress on the fetal head skull was subjected to the attraction by the vacuum extractor. This would cause the fetal head skull structure to be greatly deformed at the center of the sphere on the vacuum extractor, so there would be relatively high stress, and the stress value would be greater with an increase in vacuum pressure. In addition, when stainless steel was used as the vacuum extractor material because metals have relatively high strength, there was relatively high stress in the outer area of the vacuum extractor when pulling out the infant during delivery. In addition, when stainless steel was used as the material, the value of von Mises stress on the fetal head skull would be larger compared with using silicone rubber. The main reason is that for the load conditions, when the same displacement was applied and stainless steel was used as the vacuum extractor material, the structural deformation of the fetal head skull would be relatively large owing to the high strength and small deformation of stainless steel, so the stress value on the fetal head skull would be relatively high when using stainless steel. Therefore, previous literature indicates that using non-metallic vacuum extractors can reduce fetal skull injury [19]. The reason may be that using non-metallic vacuum extractors have relatively low stress on the head skull.

There are some limitations in finite element analysis. In this study, to evaluate the data on reaction force, among the boundary conditions, the fetal head neck was set as a fixed area, so such a setting would possibly cause the numerical results of reaction force in this study to be even larger than the actual situation. The model structures of vacuum extractors fabricated from two different materials evaluated herein were simulated with the same model structure shape, mainly owing to the need to evaluate the influences of different materials. To avoid the influences of different shapes, we herein used the same model for vacuum extractors fabricated from silicone rubber and stainless steel. In addition, the structure of fetal head model was simplified, and only two structures-scalp and skull-were established. This simplification could simply evaluate the model we want to analyze so that the research results could focus on identifying the differences in influences of the factors we want to observe and investigate.

According to the observation by finite element analysis utilized in this study, in terms of the influences of different materials of vacuum extractor and different attractive pressures on the fetal head, the results herein revealed that vacuum extractors manufactured from stainless steel would produce a relatively larger reaction force and exert relatively higher stress on the skull of fetal head. Although there are some differences between the values obtained by the analysis hereupon and the clinical situation of actual delivery, this result can more clearly reflect the trend in the real conditions, and the design of vacuum extractor can be further evaluated and investigated in the future. The results of this study can provide a biomechanical basis for obstetricians and gynecologists in choosing vacuum extractors and applying extraction force to reduce and avoid major complications and comorbidities caused by using these instruments during adaptation of fetal head to the birth canal during the process of operative delivery, thereby improving the overall quality of delivery.

\section{Conclusions}


In this study, finite element analysis was used for biomechanical interpretation of the influences of different vacuum extractor materials and different vacuum pressures on the fetal head during vacuumassisted delivery. The results showed that when relatively higher vacuum pressure was applied, the fetal head would be exposed to relatively higher stress but only slightly be affected. In addition, using vacuum extractors fabricated from materials (stainless steel) with a higher Young's modulus would cause the fetal head to be subjected to larger reaction force and stress. Therefore, according to the results via finite element analysis, we therefore suggest that clinical obstetricians can choose vacuum extractors made of non-metallic, relatively soft or and elastic materials such as silicone rubber to avoid complications during the whole process of vacuum assisted delivery such as scalp scratch, cephalohematoma, deformation of the fetal head and even intracerebral hemorrhage caused by high external force while simultaneously improving the safety and quality for medical care of mother, fetus or newborn in the entire process of operative vaginal delivery.

\section{Declarations}

\section{Funding}

Not applicable.

\section{Conflicts}

of interest/Competing interests

\section{Authors' contributions}

Conception and design, YH Chen and KM Su; methodology, KC Su and MT Tsai; data curation, KM Su, CK Lin and CC Chang; manuscript writing, YH Chen, KM Su and KC Su. All authors reviewed the manuscript.

\section{Acknowledgement}

We would like to thank Taichung Veterans General Hospital and Hung Kuang University (TCVGHHK1088001 and TCVGH-HK1098002) in Taiwan and the 3D Printing Research and Development Group of Taichung Veterans General Hospital for building the simulation computer model of this study. This research was supported and funded in part by the following grants from the Tri-Service General Hospital (TSGH-D-110137).

\section{References}

1. Cunningham, F. G., Leveno, K. J., Bloom, S. L., Dashe, J. S., Spong, C. Y., Hoffman, B. L., et al. (2018). Williams Obstetrics, 25e. McGraw Hill Medical. 
2. Gurol-Urganci, I., Cromwell, D., Edozien, L., Mahmood, T., Adams, E., Richmond, D., et al. (2013). Thirdand fourth-degree perineal tears among primiparous women in England between 2000 and 2012: time trends and risk factors. BJOG: An International Journal of Obstetrics \& Gynaecology, 120(12), 1516-1525. https://doi.org/10.1111/1471-0528.12363

3. Simonson, C., Barlow, P., Dehennin, N., Sphel, M., Toppet, V., Murillo, D., et al. (2007). Neonatal Complications of Vacuum-Assisted Delivery. Obstetrics \& Gynecology, 109(3), 626-633. https://doi.org/10.1097/01.AOG.0000255981.86303.2b

4. Johanson, R., \& Menon, V. (2000). Soft versus rigid vacuum extractor cups for assisted vaginal delivery. Cochrane Database of Systematic Reviews. https://doi.org/10.1002/14651858.CD000446

5. Ali, U. A., \& Norwitz, E. R. (2009). Vacuum-assisted vaginal delivery. Reviews in Obstetrics \& Gynecology, 2(1), 5-17.

6. O'Mahony, F., Hofmeyr, G. J., \& Menon, V. (2010). Choice of instruments for assisted vaginal delivery. Cochrane database of systematic reviews, 10(11), CD005455.

https://doi.org/10.1002/14651858.CD005455.pub2

7. Suwannachat, B., Laopaiboon, M., Tonmat, S., Siriwachirachai, T., Teerapong, S., Winiyakul, N., et al. (2011). Rapid versus stepwise application of negative pressure in vacuum extraction-assisted vaginal delivery: a multicentre randomised controlled non-inferiority trial. BJOG: An International Journal of Obstetrics \& Gynaecology, 118(10), 1247-1252. https://doi.org/10.1111/j.14710528.2011.02992.x

8. Parente, M. P., Natal Jorge, R. M., Mascarenhas, T., Fernandes, A. A., \& Silva-Filho, A. L. (2010). Computational modeling approach to study the effects of fetal head flexion during vaginal delivery. American Journal of Obstetrics \& Gynecology, 203(3), 217.e1-217.e6.

https://doi.org/10.1016/j.ajog.2010.03.038

9. Lapeer, R., \& Prager, R. (2001). Fetal head moulding: Finite element analysis of a fetal skull subjected to uterine pressures during the first stage of labour. Journal of biomechanics, 34(9), 1125-1133. https://doi.org/10.1016/S0021-9290(01)00070-7

10. Lapeer, R., Gerikhanov, Z., \& Audinis, V. A computer-based simulation of vacuum extraction during childbirth. SIMULIA Regional User Meeting RUM 2014, 2014, from https://ueaeprints.uea.ac.uk/id/eprint/56987/1/lapeer.pdf

11. Su, K.M., Yu, M.H., Su, H.Y., Wang, Y.C., \& Su, K.C. (2016). Investigating biomechanics of different materials and angles of blades of forceps for operative delivery by finite element analysis. Journal of Mechanics in Medicine and Biology, 16(04), 1650046. https://doi.org/10.1142/S0219519416500469

12. Sullivan, C., \& Hayman, R. (2008). Instrumental vaginal delivery. Obstetrics, Gynaecology \& Reproductive Medicine, 18(4), 99-105. https://doi.org/10.1016/j.ogrm.2008.01.008

13. Chen, D. W., Lin, C.L., Hu, C.C., Wu, J.W., \& Lee, M. S. (2012). Finite element analysis of different repair methods of Vancouver B1 periprosthetic fractures after total hip arthroplasty. Injury, 43(7), 10611065. https://doi.org/10.1016/j.injury.2012.01.015 
14. Raul, J.S., Baumgartner, D., Willinger, R., \& Ludes, B. (2006). Finite element modelling of human head injuries caused by a fall. International Journal of Legal Medicine, 120(4), 212-218.

https://doi.org/10.1007/s00414-005-0018-1

15. Roth, S., Raul, J.S., Ludes, B., \& Willinger, R. (2007). Finite element analysis of impact and shaking inflicted to a child. International Journal of Legal Medicine, 121(3), 223-228.

https://doi.org/10.1007/s00414-006-0129-3

16. Lin, C.L., Chang, Y.H., \& Liu, P.R. (2008). Multi-factorial analysis of a cusp-replacing adhesive premolar restoration: a finite element study. Journal of dentistry, 36(3), 194-203.

https://doi.org/10.1016/j.jdent.2007.11.016

17. Gere, J. M., \& Goodno, B. J. (2001). Mechanics of Materials 5th. Brooks Cole.

18. Enderle, J. (2012). Introduction to biomedical engineering. Academic press.

19. D’Antona, A., Mottet, N., Lenoir, P., Toubin, C., Bourtembourg, A., Ramanah, R., \& Riethmuller, D. (2020). Preliminary Study Assessing the Efficiency of a New Singleuse Obstetrical Vacuum Device: Icup2 ${ }^{\circledR}$. Archives of Obstetrics and Gynaecology, 1(2), 45-51.

\section{Figures}
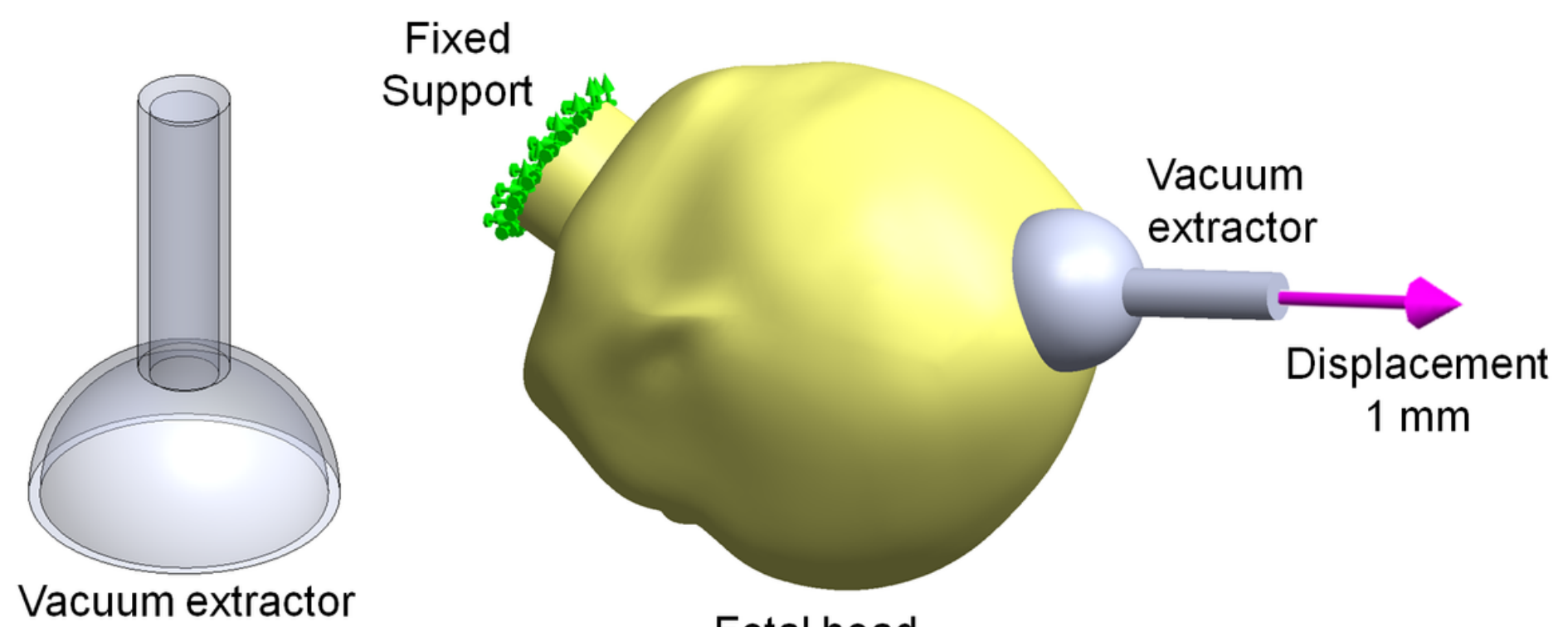

Fetal head

\section{Figure 1}

The vacuum extractor plotted herein and the finite element analysis model of using a vacuum extractor on the fetal head. 


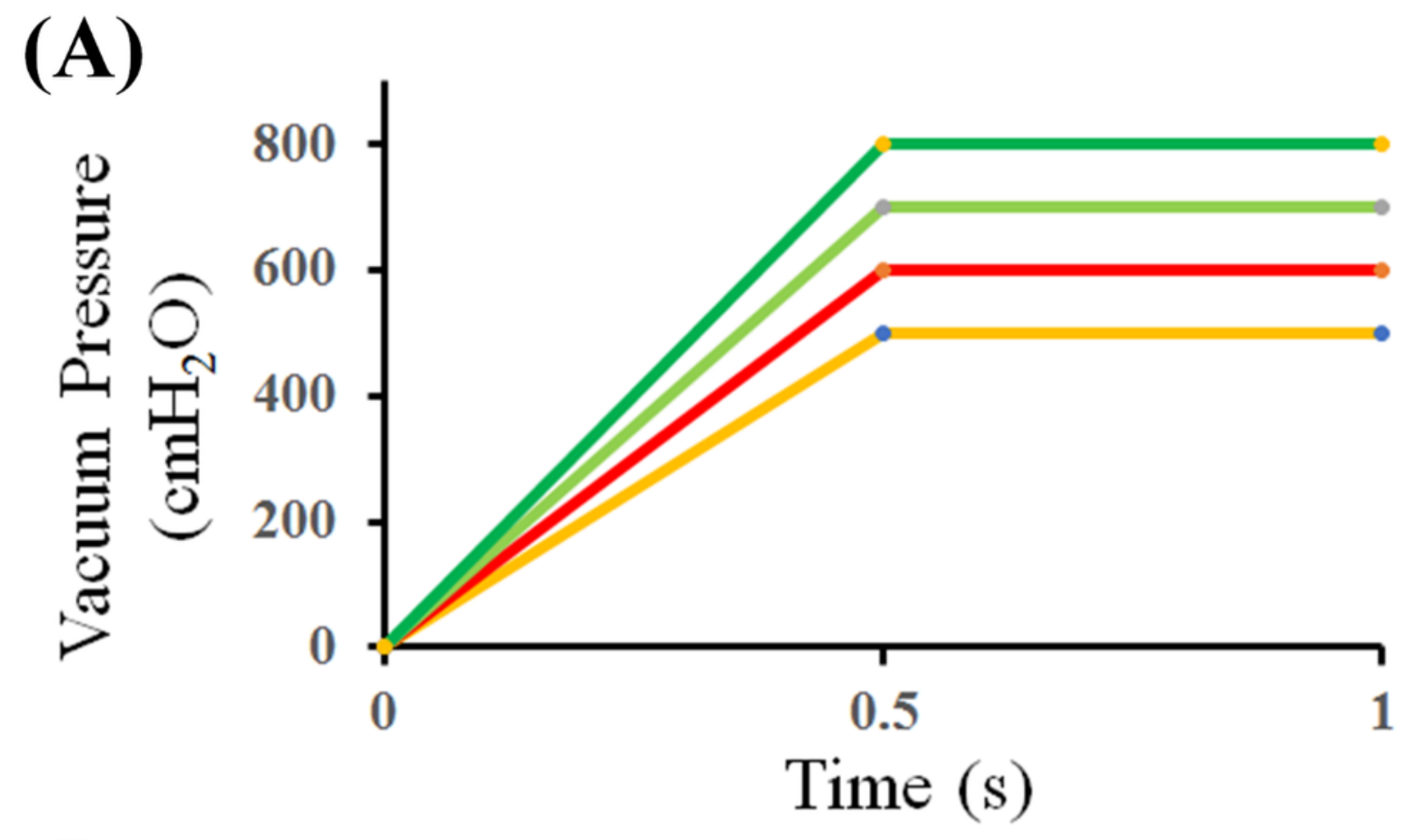

(B)

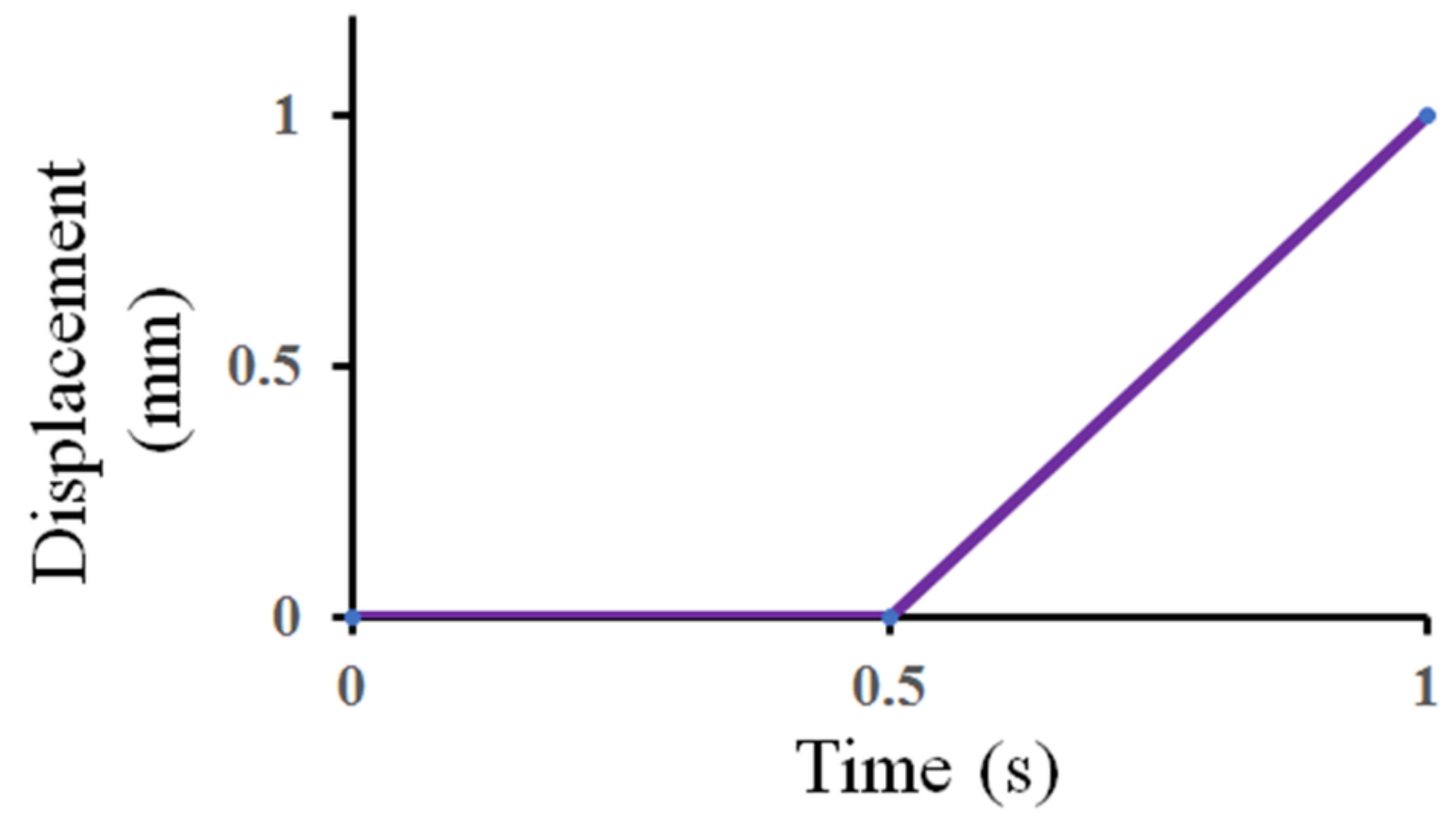

Figure 2

Magnitude of load conditions used in this study. (a) Vacuum pressure applied to the crown area of fetal head. (b) Amount of displacement applied to the vacuum extractor. 


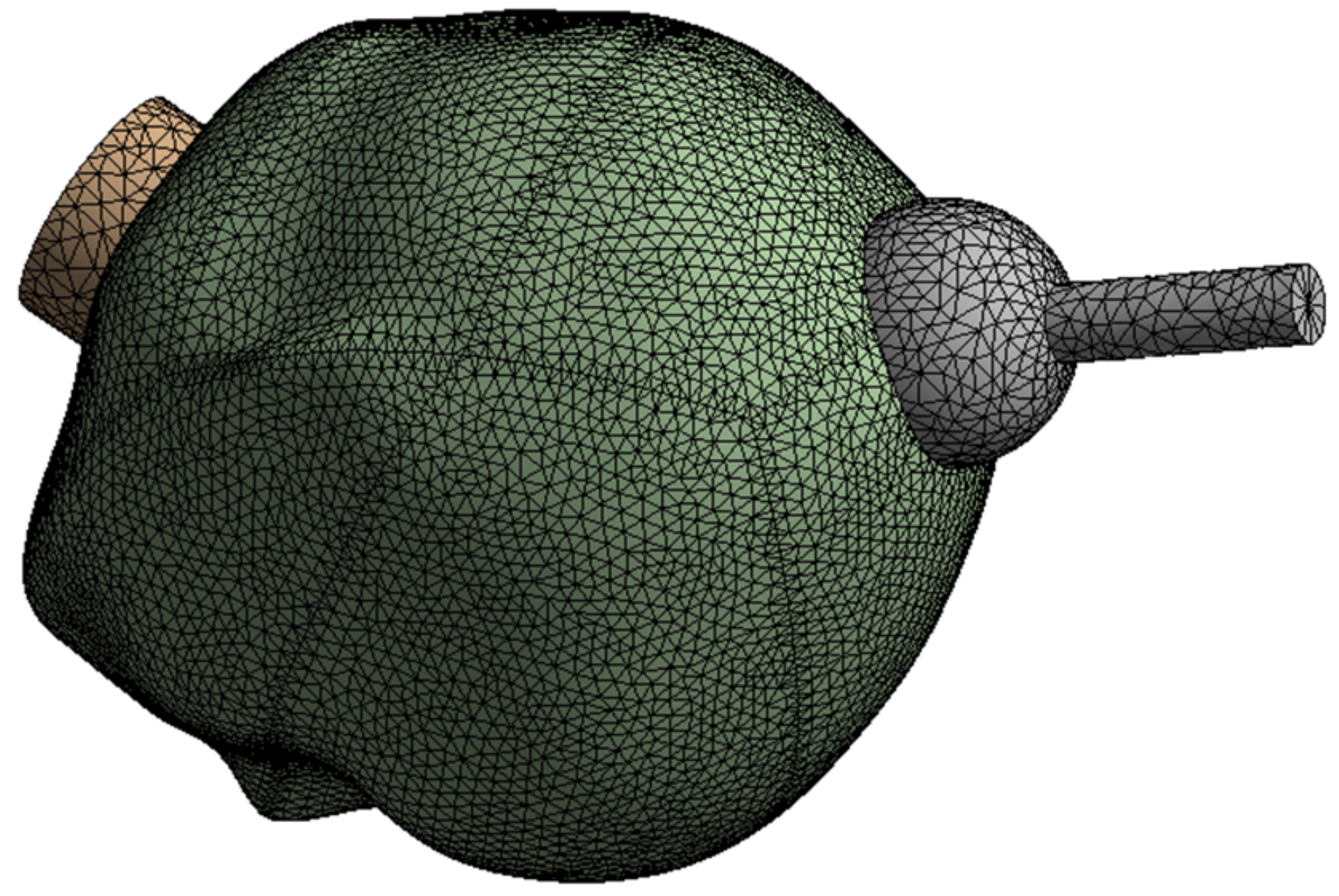

Number of mesh nodes: 131,198 Number of mesh elements: 65,270

Figure 3

Mesh model, which is the finite element analysis model used herein. 


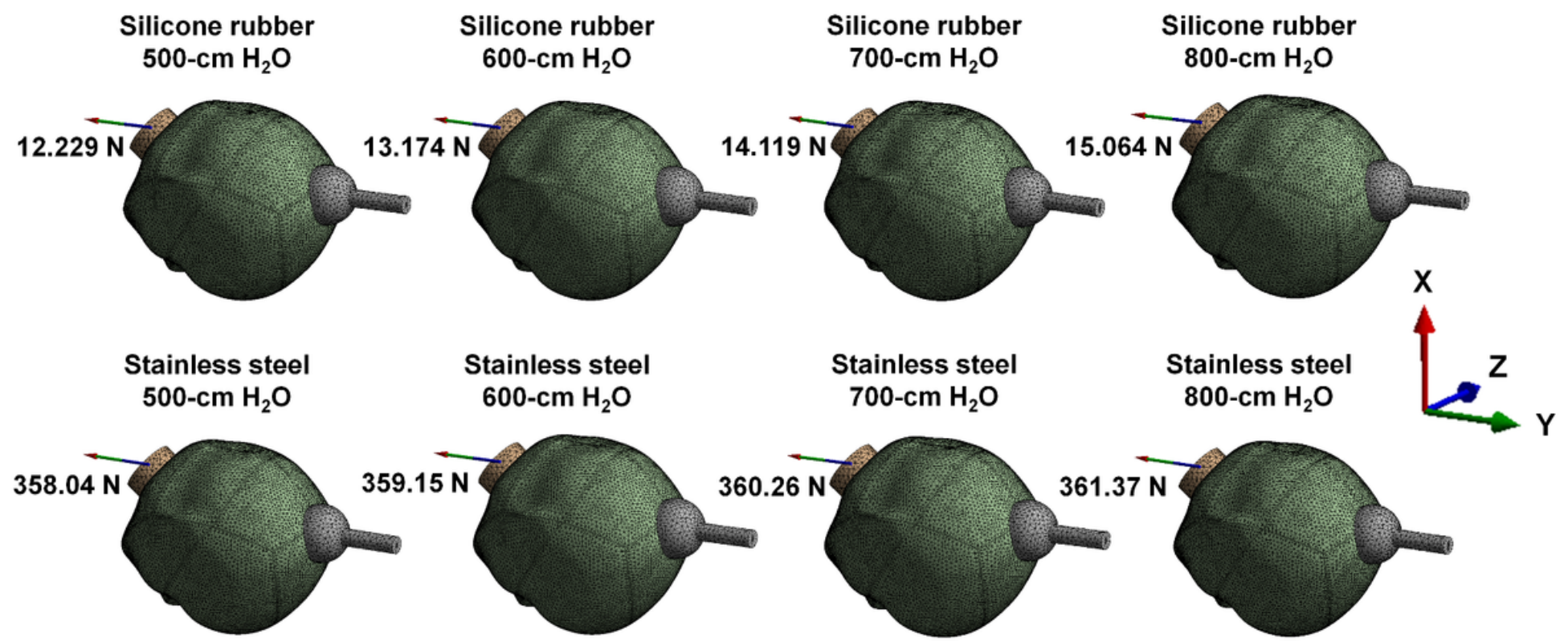

Figure 4

Direction of reaction force on the fetal head.

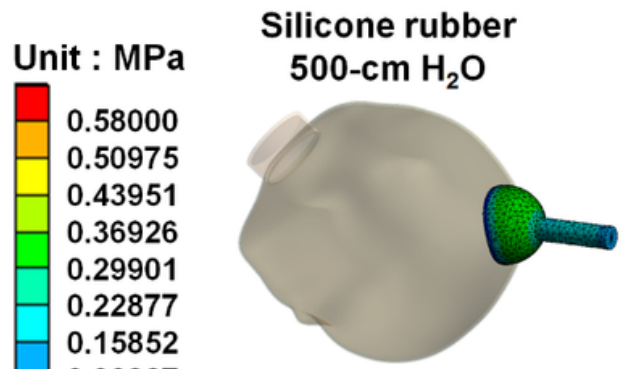

Max: $0.5858 \mathrm{MPa}$

0.06827

0.00000

Unit : MPa

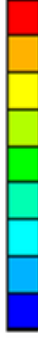

47.500

41.582

35.663

29.745

23.826

17.908

11.989

6.071

0.000

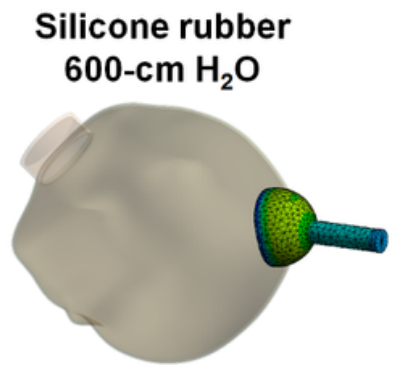

Max: $0.7066 \mathrm{MPa}$

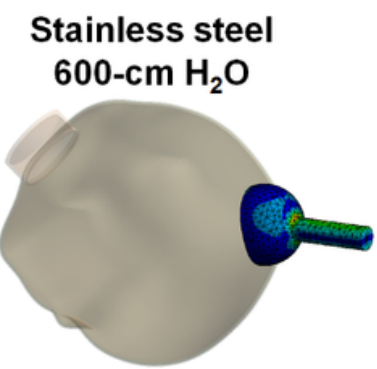

Max: 47.806 MPa

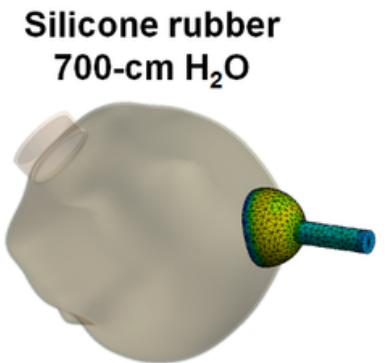

Max: $0.8285 \mathrm{MPa}$

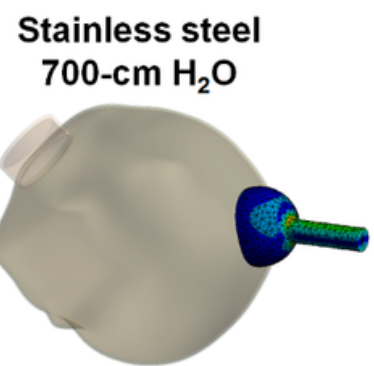

Max: $48.096 \mathrm{MPa}$
Silicone rubber 800-cm $\mathrm{H}_{2} \mathrm{O}$

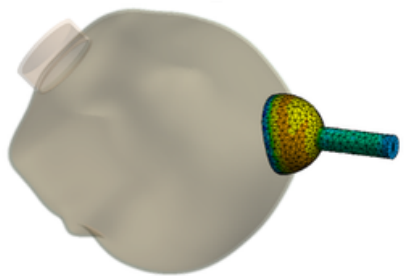

Max: $0.9510 \mathrm{MPa}$
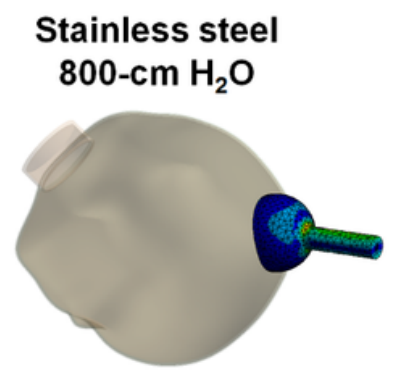

Max: $48.385 \mathrm{MPa}$

Figure 5

Distribution of von Mises stress on the vacuum extractor. 


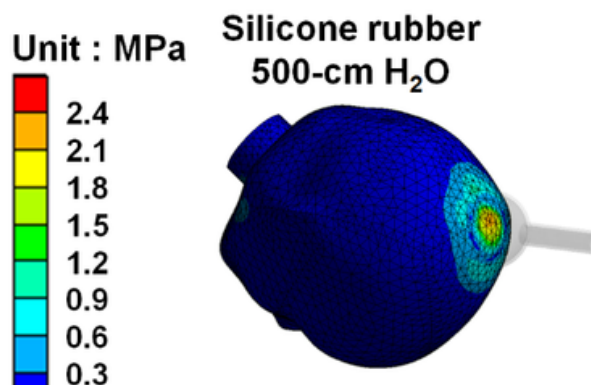

Max: $2.4755 \mathrm{MPa}$



Max: $13.547 \mathrm{MPa}$

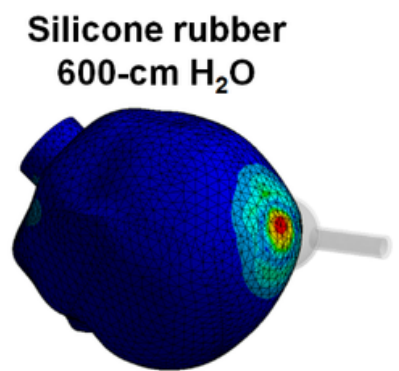

Max: $2.9889 \mathrm{MPa}$

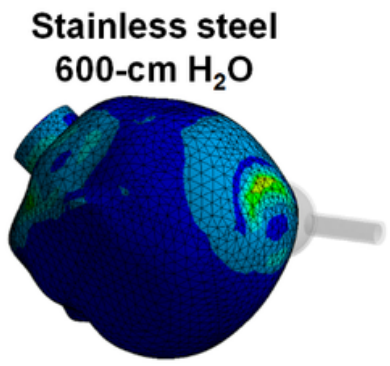

Max: $13.589 \mathrm{MPa}$

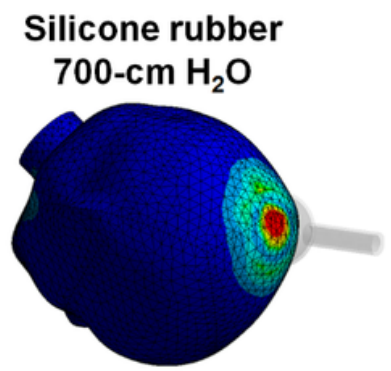

Max: $3.5022 \mathrm{MPa}$

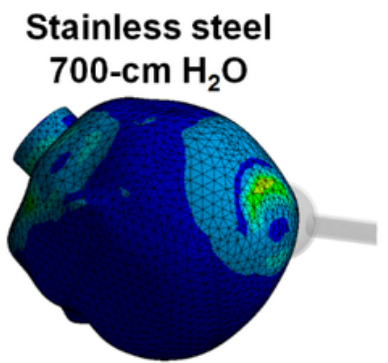

Max: $13.632 \mathrm{MPa}$

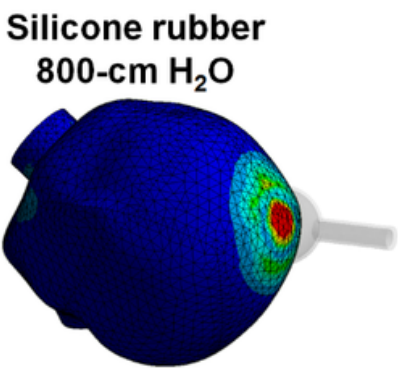

Max: $4.0156 \mathrm{MPa}$

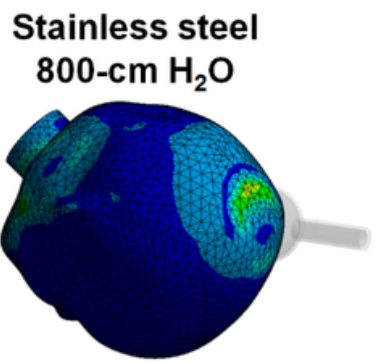

Max: $13.675 \mathrm{MPa}$

Figure 6

Distribution of von Mises stress on the skull in each material texture group. 